\title{
App Review Series: Radiology Pocket Game
}

\author{
V. B. Surya Prasath ${ }^{1}$
}

Published online: 14 November 2016

(C) Society for Imaging Informatics in Medicine 2016

\section{App Specs}

App Name: Radiology Pocket Game

App Icon URL: https://itunes.apple. com/us/app/radiologypocketgame/id968374068

App Developer: Giovanni Rinaldi

A p p Developer Website: http://www. radiologypocketgame.com/

App Price: Free

Apple App Store URL: https://itunes.apple. com/us/app/radiologypocketgame/id968374068

Google Play Store URL: https://play.google. com/store/apps/details?id=rpg.webmt.it

Category: learning, radiological anatomy, educational game

Tags: educational, free, radiology, anatomy, iPadcompatible

Works Offline: No

FDA Approval: NA

Quick Review (1 star, lowest; 5 stars, highest)

Overall Rating (1-5): 3.5

Content (1-5): 3.5

Usability (1-5): 3.5

Pros: Quick testing of image-based radiological anatomy quizzes to test your knowledge and learn. Easy to learn and use, and the instructions are clear.

Cons: Limited number of test cases. Some of the answers are found by scrolling down the option list, which increases your reaction times.

V. B. Surya Prasath prasaths@missouri.edu

1 Computational Imaging and VisAnalysis (CIVA) Lab, Department of Computer Science, University of Missouri, Columbia, MO 65211, USA
At a glance: If you like some simple way to learn radiological anatomy, then this quiz is for you.

A simple interface allows you to see the images and lets you to choose an answer from a list below.

\section{Introduction}

Radiology Pocket Game (RPG in short) is an image-based radiological anatomy quiz app. This app is a fun way to quickly learn some major anatomical structures in a game-based environment. As the RPG makes use of the radiological images (conventional radiology, computed tomography, and magnetic resonance imaging) and major anatomical structures marked together, it provides a unique way of learning and keeps track of correct answers as well as the average reaction time.

\section{Purpose/Features/Content}

The purpose of this RPG app is to teach the user's anatomy using radiological images. The user is required to sign in and create a username and password or can Login with Facebook account. After Login, the user can start playing. To start the play, the user can choose one or more of the pre-defined categories: "Chest," "Cardiovascular," "MSK" (musculoskeletal), "Abdomen," and "Neuroradiology," or can choose to "Play random"; see Fig. 1.

The main features of the RPG app are (1) the use of radiological images with marked (identified by lines, encircled, arrows) anatomical landmarks along with multiple answers which are presented to the user in a random fashion, (2) the vibration and red font indicate if the user has selected a wrong answer and green font indicates the right answer, and (3) 

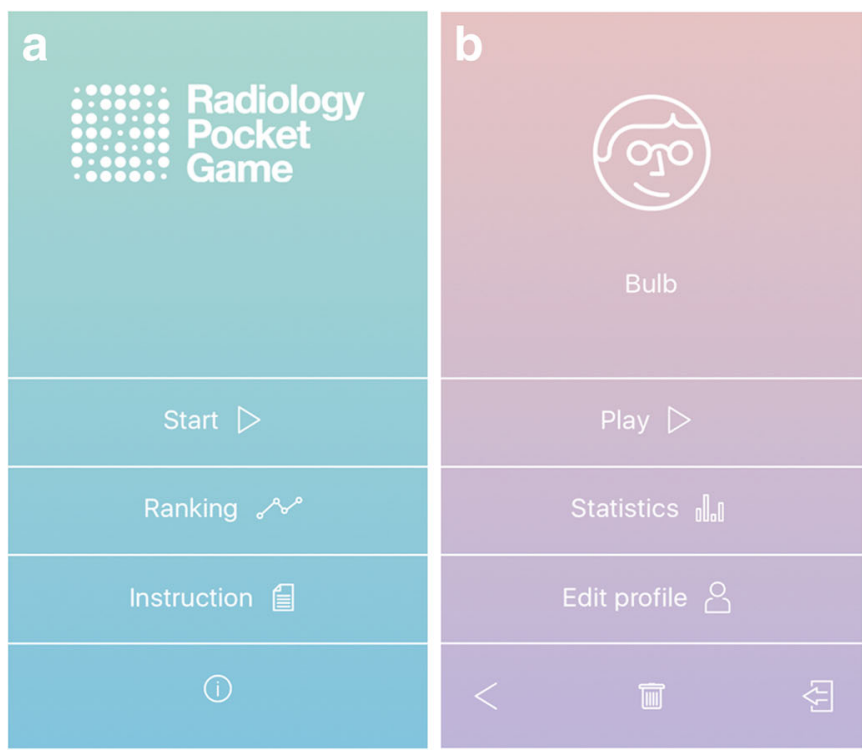

Fig. 1 Radiology Pocket Game consists of radiological image-based quizzes on anatomical landmarks. a Initial homepage when the app is opened. b After signing in, the user can tap "Play" or check his/her "Statistics" or "Edit profile." c When "Play" resumes, the user is

overall reaction time, number of correct answers, and percentage $(\%)$ of precision are given after each play. There is also an overall statistics for the user based on all the total games played so far indicating total points with how many correct/ wrong answers, percentage of precision, and average reaction time given. The user can share each play scores in social media Twitter and Facebook.

The main contents consist of clear and good quality radiological images (obtained from open-source websites such as Radiopaedia.org and Wikimedia Commons) with anatomical regions identified, encircled, or pointed out by an arrow and questions about them.

\section{Usability}

The RPG app is simple to use requiring only single finger taps to select options and play the games. When playing, the user can simply tap on the answer and the app buzzes (if vibration on the smartphone/tablet is enabled) if it is a wrong answer and moves on to the next question. After a set of random questions (usually less than six questions), the RPG app

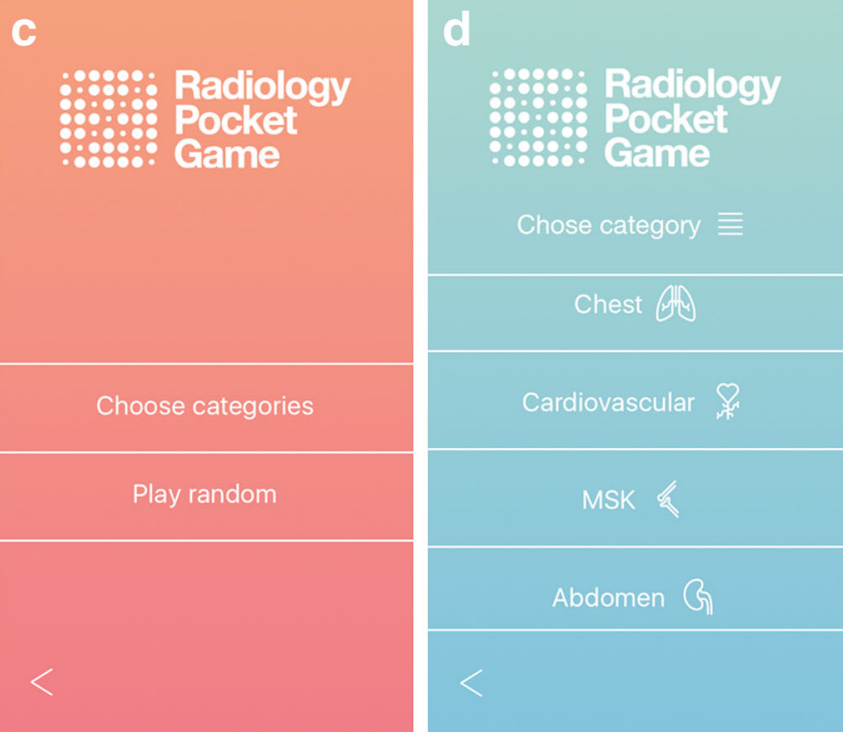

presented with two choices, "Choose categories" or "Play random." d Under "Choose categories," the user is presented with "Chest," "Cardiovascular," "MSK," "Abdomen," and "Neuroradiology." The user can select multiple categories to play

shows a statistics screen where the user can see his progress achieved so far, see Fig. 2 for examples.

\section{Good}

Relatively easy game setup with image on top and corresponding multiple answer options given immediately below. Users need only to select (tap) an option, and the game moves to the next question automatically. Good image quality.

Room for Improvement

Requires scrolling down to see the multiple choice answers; thus, the reaction time taken to answer is affected, see Fig. 3 for example. Here, the options are "Right lower zone" and "Left middle zone" are visible, though the other options "Left costophrenic angle" and "Left costophrenic recess" are not visible, with "Left costophrenic angle" being the correct answer is visible only after scrolling down.

You cannot quit or pause the game when the series of questions are presented.

Limited number of images and anatomical structures in each category. 
a

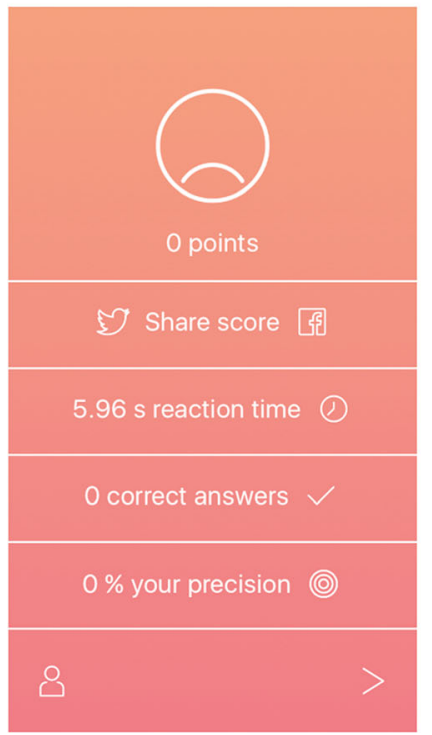

b

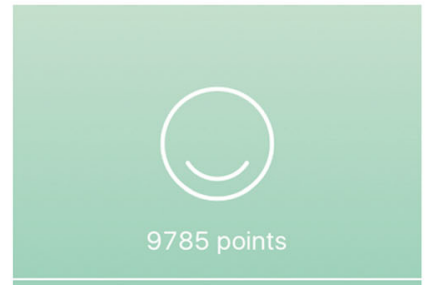

Share score

1.06 s reaction time (1)

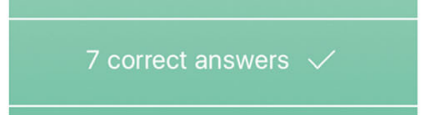

$70 \%$ your precision (0)

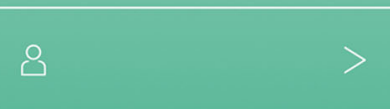

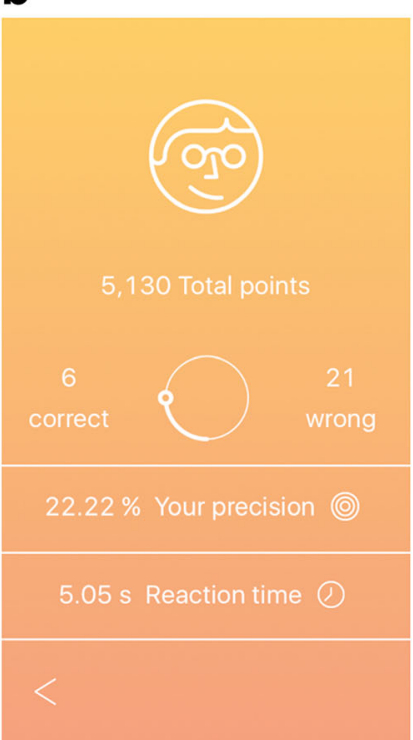

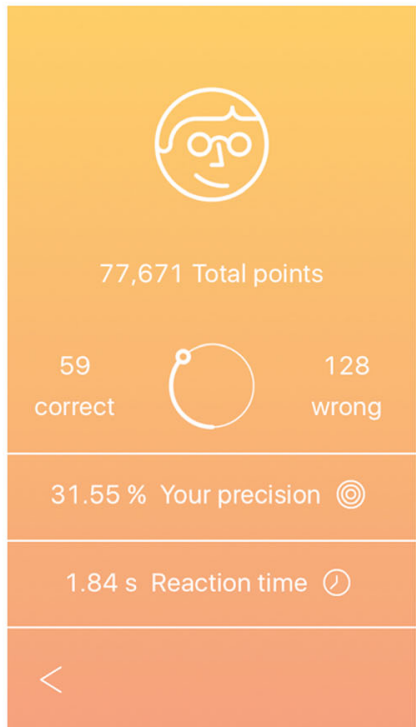

Fig. 2 Radiology Pocket Game keeps track of the reaction time (average time taken to answer each question), how many correct answers are given, and percentage of precision. Two examples of $\mathbf{a}$ final report card after each play and $\mathbf{b}$ overall user statistics

a

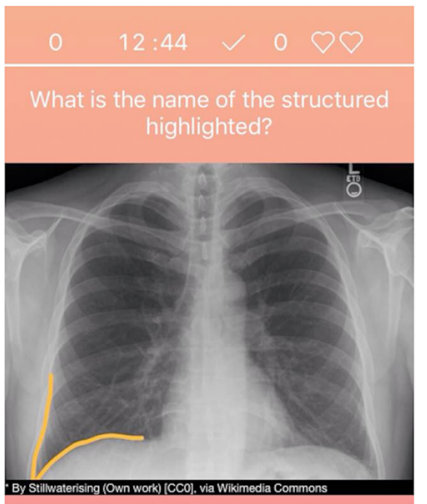

Right lower zone

Left middle zone

Fig. 3 Radiology Pocket Game has multiple choice-based questions by presenting a radiological image and identifying an anatomical structure. Two examples with chest X-ray with structures highlighted, further

Right lower zone

Left middle zone

Left costophrenic angle

Left costophrenic recess b
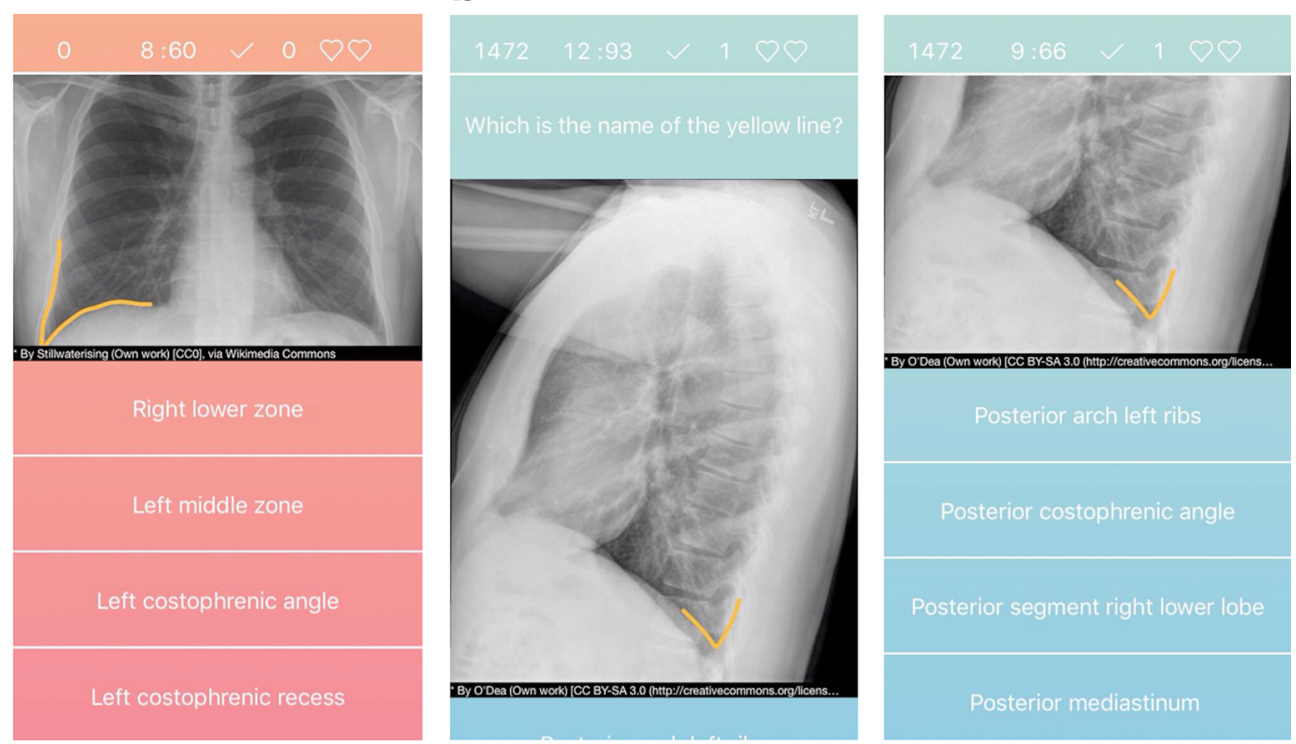

answer options after scrolling down. a Left costophrenic angle. b Posterior costophrenic angle 